\title{
SHARP ESTIMATES FOR $m$-LINEAR $p$-ADIC HARDY AND HARDY-LITTLEWOOD-PÓLYA OPERATORS ON $p$-ADIC CENTRAL MORREY SPACES
}

\section{YANGKENDI DENG, DUNYAN YAN AND MingQUAN WEI*}

Abstract. In this paper, we consider the mapping property of both $m$-linear $p$-adic Hardy operator $\mathscr{H}_{m}^{p}$ and Hardy-Littlewood-Pólya operators $T_{m}^{p}$ on $p$-adic central Morrey-type spaces $B^{p_{1}, \lambda_{1}}\left(\mathbb{Q}_{p}^{n}\right) \times \cdots \times B^{p_{m}, \lambda_{m}}\left(\mathbb{Q}_{p}^{n}\right)$. The obtained bounds turn to be sharp when $\lambda_{1} p_{1}=\cdots=\lambda_{m} p_{m}$.

Mathematics subject classification (2020): 26A33, 42B25, 42B35.

Keywords and phrases: Hardy operator, Hardy-Littlewood-Pólya operator, $p$-adic fields, $p$-adic central Morrey spaces, sharp bound.

\section{REFERENCES}

[1] Josefina Alvarez, Joseph Lakey, and Martha Guzmán-Partida, Spaces of bounded $\lambda$ central mean oscillation, Morrey spaces, and $\lambda$-central Carleson measures, Collect. Math. 51 (2000), no. $1,1-47$.

[2] Amjad Hussain, Naqash Sarfraz, And Ferit Gurbuz, Sharp weak bounds for p-adic hardy operators on p-adic linear spaces, 2020.

[3] ÁRPÁd BÉNYI AND CHOONGHONG OH, Best constants for certain multilinear integral operators, J. Inequal. Appl. (2006), Art. ID 28582, 12.

[4] Michael Christ And Loukas Grafakos, Best constants for two nonconvolution inequalities, Proc. Amer. Math. Soc. 123 (1995), no. 6, 1687-1693.

[5] William G. Faris, Weak Lebesgue spaces and quantum mechanical binding, Duke Math. J. 43 (1976), no. 2, 365-373.

[6] Zunwei Fu, Qing Yan Wu, and ShanZhen Lu, Sharp estimates of p-adic Hardy and HardyLittlewood-Pólya operators, Acta Math. Sin. (Engl. Ser.) 29 (2013), no. 1, 137-150.

[7] Zunwei Fu, Loukas Grafakos, Shanzhen Lu, and Fayou ZhaO, Sharp bounds for $m$-linear Hardy and Hilbert operators, Houston J. Math. 38 (2012), no. 1, 225-244.

[8] G. H. HARDY, Note on a theorem of Hilbert, Math. Z. 6 (1920), no. 3-4, 314-317.

[9] AmJad Hussain And NaqASh SARFraZ, Optimal weak type estimates for p-adic Hardy operators, $p$-Adic Numbers Ultrametric Anal. Appl. 12 (2020), no. 1, 29-38.

[10] Wenjuan Li, QIngying Xue, And KôZÔ Yabuta, Maximal operator for multilinear CalderónZygmund singular integral operators on weighted Hardy spaces, J. Math. Anal. Appl. 373 (2011), no. 2, 384-392.

[11] NAQASH SARFRAZ AND FerIT GURBUZ, Weak and strong boundedness for p-adic fractional hausdorff operator and its commutator, 2019.

[12] M. H. Taibleson, Fourier analysis on local fields, Princeton University Press, Princeton, N.J.; University of Tokyo Press, Tokyo, 1975.

[13] V. S. Vladimirov and I. V. Volovich, p-adic quantum mechanics, Comm. Math. Phys. 123 (1989), no. 4, 659-676.

[14] V. S. Vladimirov, I. V. Volovich, And E. I. Zelenov, p-adic analysis and mathematical physics, Series on Soviet and East European Mathematics, vol. 1, World Scientific Publishing Co., Inc., River Edge, NJ, 1994. 
[15] Qingyan Wu And Zunwei Fu, Sharp estimates of $m$-linear p-adic Hardy and Hardy-LittlewoodPólya operators, J. Appl. Math. (2011), Art. ID 472176, 20.

[16] Minghua Yang, Zunwei Fu, And Jiny I Sun, Existence and large time behavior to coupled chemotaxis-fluid equations in Besov-Morrey spaces, J. Differential Equations 266 (2019), no. 9, 58675894. 